\title{
An unusual story of electrical storm on the graft.
}

\author{
etienne charbonneau ${ }^{1}$, Bernard Lelong ${ }^{2}$, celine chabanne ${ }^{1}$, vincent galand ${ }^{1}$, and Erwan \\ Flecher $^{2}$ \\ ${ }^{1}$ CHU Pontchaillou \\ ${ }^{2}$ Pontchaillou Hospital
}

November 30, 2020

\begin{abstract}
Background: Cancers, and specifically lymphomas, are one of the main causes of morbidity and mortality after heart transplant. We encountered this complication on a 54-year-old male patient, which brought to our attention the lack of a specific and appropriate treatment for a primary cardiac lymphoma on the graft. Objective: This case report highlights the challenging management of primitive heart transplant lymphoma and its treatment. Method: electronic medical record literature reviewed through Pubmed interface of Medline. Result: We report the case of an Epstein-Barr Virus (EBV) induced primary cardiac lymphoma on the graft. The lymphoma spread quickly in spite of the numerous treatments we tried and resulted in rhythmic complication. Endocavitary implantable cardioverter defibrillator was implanted but quickly removed due to local complications. The patient died from a sudden death at home shortly after. Conclusions: Primary cardiac lymphoma on the graft is highly rare with poor prognosis and rhythmic complications. Further studies are needed to assess the benefits of other treatment approaches, such as radiofrequency ablation techniques.
\end{abstract}

An unusual story of electrical storm on the graft.

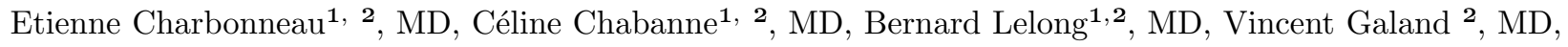
Erwan Flécher ${ }^{1}, \mathrm{MD}, \mathrm{PhD}$.

${ }^{1}$ Department of cardiothoracic and vascular surgery, Rennes University Hospital, Rennes, France

${ }^{2}$ Department of cardiology, Rennes University Hospital, Rennes, France.

The data that support the findings of this study are openly available in Journal of Cardiac Surgery at http://doi.org/[doi], reference number [reference number].

Corresponding Author:

Dr Etienne Charbonneau

Departement of Cardiology

CHU Pontchaillou

2 henri Le Guilloux St

35000 Rennes, France

Tel : +33648883662

Email : echarbonneau@hotmail.fr

Declarations of interest: none 
Funding : none AbstractBackground: Cancers, and specifically lymphomas, are one of the main causes of morbidity and
mortality after heart transplant. We encountered this complication on a 54-year-old male patient, which
brought to our attention the lack of a specific and appropriate treatment for a primary cardiac lymphoma
on the graft.

Objective: This case report highlights the challenging management of primitive heart transplant lymphoma and its treatment.

Method: electronic medical record literature reviewed through Pubmed interface of Medline.

This study was approved by the CHU Rennes Ethics Committee.

Written informed consent was obtained from the patient for the publication of this case report.

Result: We report the case of an Epstein-Barr Virus (EBV) induced primary cardiac lymphoma on the graft. The lymphoma spread quickly in spite of the numerous treatments we tried and resulted in rhythmic complication. Endocavitary implantable cardioverter defibrillator was implanted but quickly removed due to local complications. The patient died from a sudden death at home shortly after.

Conclusions: Primary cardiac lymphoma on the graft is highly rare with poor prognosis and rhythmic complications. Further studies are needed to assess the benefits of other treatment approaches, such as radiofrequency ablation techniques.

Keywords: lymphoma, heart transplant, ventricular arythmia, electrical storm.

Abbreviations; ICD: implantable cardioverter defibrillator, EBV: ebstein bar virus, TV: ventricular tachycardia, CICU: Cardiac Intensive Care Unit.

\section{Introduction}

Cancers remain one of the main causes of long-term mortality after heart transplantation. According to the International Society of Heart and Lung Transplantation (ISHLT), 16\% of the transplant recipients develop a cancer within five years and almost $30 \%$ within ten years $(1,2)$. Among this population, $50 \%$ develop cutaneous cancers but lymphomas are frequent as well, representing $10 \%$ of these patients (2). Interestingly, the incidence of lymphoma does not regularly increase during follow-up but is more important during the 5 years following transplantation (3). Notably, post-cardiac transplant lymphomas are more likely extranodal with lesions of the central nervous system, lungs, or liver (3). However, isolated cardiac graft lymphomas are extremely unusual and have been poorly reported so far. We aimed at describing the case of a patient who developed an isolated graft lymphoma after receiving heart transplantation.

\section{Case report}

In 2017, a 52-year-old man was successfully transplanted for acute advanced heart failure due to massive myocardial infarction, going through a step of left ventricular assistance device complicated by thromboembolic accident. Serology was mismatched, the donor was EBV positive and the patient EBV negative. After transplant no adverse event occur except an moderate chronic renal failure. For protect the renal function we treated him by prednisolone, mycophénolate mofétil, éverolimus and ciclosporine in order to use low dose of calcineurine inhibitor. In December 2019, he was admitted to our hospital for severe but well-tolerated palpitations. The ECG showed a ventricular tachycardia (Fig.1) that was reduced by beta blocker (Atenolol (R). Echocardiographic parameters showed no myocardial dysfunction and no sign of graft rejection. Further investigations did not bring any biological abnormalities beside an Epstein-Barr Virus (EBV) high replication - $304000 \mathrm{IU} / \mathrm{ml}$. Cardiac computed tomography and cardiac magnetic resonance imaging revealed two nodular images developed in the antero-medium and apical wall of the right ventricle and extending intracavitary and to epicardial area (Fig. 1). Endomyocardial biopsies were performed and ruled out an acute rejection. Nonetheless, the microscopic appearance revealed a post-transplantation monomorphic lymphoproliferative 
disorder, EBV induced, high grade B cell lymphoma type Diffuse large B-cell lymphoma (DLBCL). As illustrated in Figure 1, the 18FDG-PET indicated an important lymphoma extension with three hypermetabolic pericardial tumors. Of note, a coronary angiography ruled out an additionnaly underlying ischemic cardiomyopathy but hypervascularization was observed in the intramyocardial tumoral masses. We proceed with an initial therapy of four cycles of rituximab and a reduction of the immunosuppressive drug in order to decrease the EBV replication. Unfortunately, despite pursing beta-blockers treatment ventricular arrhythmias still occurred including ventricular premature beats and sustained ventricular tachycardia (VT). Due to the sporadic nature of the arrhythmia, we decided not to implant an implantable cardioverter-defibrillator (ICD) as the first line therapy but to optimize the antiarrhythmic therapy by inflating beta-blockers to the maximum tolerated dose. Despite optimal medical therapy including amiodarone and beta-blockers, the patient presented an electrical storm associated with hemodynamic instability and was hospitalized in the Cardiac Intensive Care Unit (CICU). In CICU, the VT recurrences were controlled using Esmolol infusion. After 2 days, the patient was free from VT recurrence and Nadolol was introduced with a good tolerance, authorizing the continuation of the chemotherapy. Fourth cycles of Rituximab were consequently completed and a PET reevaluation was realized showing the persistence of an intense hypermetabolism in the three pericardial tumors. A new therapy using Doxorubicin, Cyclophosphamide and Vincristine was implemented as an emergency treatment with regular PET reevaluations and a minimized immunosuppression. In order to prevent the sudden cardiac death, the patient was implanted with an endocavitary ICD (Medtronic, Dublin, ireland). The first cycle of chemotherapy caused a pancytopenia with a profound thrombocytopenia resulting in the formation of an ICD pocket hematoma. The hematoma spontaneously progressed to fistula, leading to a revision surgery with device removal. Before hospital discharge, the patient received a life-vest (Zoll, Chelmsford, USA) that was badly tolerated. Successive chemotherapy cycles were ineffective with a tachyphylaxis situation and a quick progression of the tumoral lesions assessed by cardiac imaging and significant EBV replication. The patient died from a sudden death at home 3 month after the diagnosis, not wearing his life-vest anymore because of the bone pain caused by chemotherapy.

\section{Discussion}

Isolated primary cardiac lymphoma is an exceptional post-transplant complication with only very rare cases previously reported to the best of our knowlegde (4). The appropriate treatment strategy remains challenging. We decided not to propose a second heart transplantation taking account the uncertain risk of recurrence on the graft. Indeed, only few papers suggested such therapeutic option for primary cardiac tumors but with heterogeneous results $(5,6)$.

Considering the immunosuppressive strategy, we chose an immunosuppressive quadritherapy (Prednisolone, mycophénolate mofétil, éverolimus, ciclosporine), before the lymphoma, in order to use lower dose of calcineurin inhibitors. However this strategy may be associated with an increase in infectious and neoplastic complications and place and benefits of such quadritherapy remain matter of debate.

To prevent sudden death in this case, we implanted an endocavitary ICD. The arrhythmia triggering factor was obvious but its persistence and the eventuality of a post-treatment fibrosis area was uncertain. Moreover, we considered that the rhythmic risk would remain high even at a distance from the lymphoma. Radiofrequency catheter ablation might be an interesting option for such patients but the procedure seems associated with high risks including tumor spreading or ventricular hyperexcitability. Additionally, the penetration of the radiofrequency current would be probably insufficient due to the large tumoral masses. More recently few teams developed non-invasive ablation methods of the VT using external radiation (7). This procedure might have been interesting in this case as it presents the double benefit of enhanced security of the procedure and of transmural ablation that might have been beneficial to the lymphoma treatment.

\section{Conclusion :}

Primary cardiac lymphoma of the graft is a very rare complication with a very poor prognosis. Its management and treatment remain discussed in this population. Adaptation of immunosuppression and development of non-invasive radiofrequency ablation techniques might be interesting research track in the future. 


\section{References:}

1. Khush KK, Cherikh WS, Chambers DC, et al. The international thoracic organ transplant registry of the international society for heart and lung transplantation: thirty-fifth adult heart transplantation report-2018; focus theme: multiorgan transplantation. J Heart Lung Trans. 2018;37:1155. https://doi.org/10.1016/J.HEALUN.2018.07.022

2. Crespo-Leiro MG, Alonso-Pulpón L, Vázquez de Prada JA, et al. Malignancy after heart transplantation: incidence, prognosis and risk factors. Am J Transplant. 2008;8:1031-1039. https://doi.org/10.1111/j.1600-6143.2008.02196.x

3. Salla Jäämaa-Holmberg, Birgitta Salmela, Karl Lemström, Eero Pukkala \& JyriLommi. Cancer incidence and mortality after hearttransplantation - A population-based national cohort study. Acta oncologica. 2019;58:859-863 https://doi.org/10.1080/0284186X.2019.1580385

4. Burtin P, Guerci A, Boman F, Dopff C, Pinelli G, Haberer JP, Villemot JP. Malignant lymphoma in the donor heart after heart transplantation. Eur Heart J. 1993 Aug;14(8):1143-5. doi: 10.1093/eurhe$\operatorname{artj} / 14.8 .1143$.

5. Yuh DD, Kubo SH, Francis GS, Bank A, McDonald KM, Jessurun J, Verfaillie C, Shumway SJ. Primary cardiac lymphoma treated with orthotopic heart transplantation: a case report. J Heart Lung Transplant. 1994 May-Jun;13(3):538-42. PMID: 8061032.

6. Gowdamarajan A, Michler RE. Therapy for primary cardiac tumors: is there a role for heart transplantation? Curr Opin Cardiol. 2000 Mar;15(2):121-5. doi: 10.1097/00001573-200003000-00010.

7. Cuculich PS, Schill MR, Kashani R, Mutic S, Lang A, Cooper D, Faddis M, Gleva M, Noheria A, Smith TW, Hallahan D, Rudy Y, Robinson CG. Noninvasive Cardiac Radiation for Ablation of Ventricular Tachycardia. N Engl J Med. 2017 Dec 14;377(24):2325-2336. doi: 10.1056/NEJMoa1613773.

\section{Hosted file}

Figure 1 whithout legend.pdf available at https://authorea.com/users/380017/articles/496153an-unusual-story-of-electrical-storm-on-the-graft 